Wilfrid Laurier University

Scholars Commons @ Laurier

Physics and Computer Science Faculty

Publications

Physics and Computer Science

1987

\title{
Subharmonic Locking in Josephson Weak Links
}

G.W. Frank

University of Western Ontario

A.S. Deakin

University of Western Ontario

M.A.H. Nerenberg

University of Western Ontario

James A. Blackburn

Wilfrid Laurier University, jabjabjab@cogeco.ca

Follow this and additional works at: https://scholars.wlu.ca/phys_faculty

\section{Recommended Citation}

Frank, G.W.; Deakin, A.S.; Nerenberg, M.A.H.; and Blackburn, James A., "Subharmonic Locking in Josephson Weak Links" (1987). Physics and Computer Science Faculty Publications. 53.

https://scholars.wlu.ca/phys_faculty/53

This Article is brought to you for free and open access by the Physics and Computer Science at Scholars Commons @ Laurier. It has been accepted for inclusion in Physics and Computer Science Faculty Publications by an authorized administrator of Scholars Commons @ Laurier. For more information, please contact scholarscommons@wlu.ca. 


\title{
Subharmonic locking in Josephson weak links
}

\author{
G. W. Frank, A. S. Deakin, and M. A. H. Nerenberg \\ Department of Applied Mathematics, University of Western Ontario, London, Ontario, Canada N6A $5 B 9$ \\ James A. Blackburn \\ Department of Physics, Wilfred Laurier University, Waterloo, Ontario, Canada \\ (Received 11 August 1986; revised manuscript received 4 November 1986)
}

\begin{abstract}
After some controversy, it has been shown that subharmonic voltage (phase) locking does not exist in the ac-driven overdamped resistively shunted junction model of a Josephson weak link. We predict that for a very similar system of a pair of coupled links without ac drive, mutual subharmonic locking can take place. We demonstrate our thesis both by a careful numerical simulation of the exact equations of the model and by a second-order analytical perturbation calculation based on the coupling parameter.
\end{abstract}

\section{INTRODUCTION}

It is now generally accepted that the resistively shunted junction (RSJ) model, with capacitance set to zero, has turned out to be adequate in predicting the principal behavior of Josephson weak links. ${ }^{1}$ is

For a single link the corresponding differential equation

$$
I=I_{c} \sin \phi+\frac{1}{R_{N}} \frac{\hbar}{2 e} \frac{d \phi}{d t},
$$

where $I=I_{\mathrm{dc}}+I_{\mathrm{ac}}$ is the sum of both supercurrent and normal (quasiparticle) current through the junction, $R_{N}$ is the resistance of the quasiparticles, $I_{c}$ is the critical current of the weak link, while $\phi$ is the phase difference across it.

Despite the apparent simplicity of this equation there has been controversy in the history of its use. Early numerical work ${ }^{2}$ seemed to indicate the existence of $s u b$ harmonic dc voltage locking of the junction to the frequency of an external ac current source, along with voltage locking and harmonic locking. Subsequent analytical work has proven rigorously that the subharmonic type of locking with its corresponding voltage steps cannot occur in this model. ${ }^{3,4}$ This contrasts with the more elaborate RSJ model in which one presumes that the junction has capacitance as well as resistance. There, subharmonic locking is predicted. ${ }^{5}$ The overdamped (noncapacitive) RSJ model contrasts further with the more elaborate model by having, even when ac driven, no chaotic solutions, a consequence of its two-dimensional phase space. ${ }^{6,7}$ It has by now been very amply demonstrated that the capacitive model supports chaos when driven by an ac current source. $^{8}$

Since a weak link itself can act as a source of ac current, the system of two dc-biased weak links, coupled to each other via some mechanism ${ }^{1}$ should bear strong resemblance to the ac- and dc-biased single weak link. Of course, the weak links' mutual interaction distinguishes it from the one-way action of an ac source upon a single link.
It has been shown ${ }^{9-12}$ that the overdamped RSJ model successfully describes many of the phenomena actually observed experimentally for a coupled system. This includes mutual equal-voltage locking, harmonic-voltage locking, as well as coherent effects outside locking zones. It also predicts the possibility of mutual-voltage locking of an indefinitely large number of such links which would lead them to oscillate and radiate coherently. ${ }^{13}$ A review of experimental and theoretical work on mutual equalvoltage locking was published a few year ago. ${ }^{14}$

The question addressed here is whether a pair of coupled noncapacitive Josephson weak links can mutually lock subharmonically; that is, given two positive relatively prime integers $n_{1}, n_{2}$, neither of which is unity, whether there is a region in parameter space of nonzero measure such that $n_{1} V_{1}=n_{2} V_{2}$, where $V_{1}$ and $V_{2}$ are the average voltages of the individual junctions. To the best of our knowledge, for such a system, this type of locking has not yet been observed experimentally.

We approach the problem from two virtually independent angles. The lesson to be learned from the original mistaken positive result ${ }^{2}$ for the ac-driven single link is that numerical simulation of the equations alone might not be an adequate test for this question. Numerical approximation of the differential equation itself might lead to an effective spurious $\cos \phi$ term, ${ }^{4}$ or possibly some other defect. In addition, apparent locking for long periods may be observed, only to be followed by slippage if the simulation is allowed to continue long enough.

We therefore carried out a complementary analytical perturbation expansion in order to corroborate the numerical simulation. This had the added benefit of illuminating the qualitative behavior of the system in the region of subharmonic locking. In particular this calculation showed that the latter, unlike equal-voltage locking and harmonic locking, cannot appear to first order in the coupling constant $\alpha$; perhaps a vestige of its nonexistence for the ac-driven single link. This might explain in part why subharmonic locking in this system has remained unobserved, ${ }^{10}$ since for the most part experimental $\alpha$ values have been modest. 
TABLE I. A summary of the results on the locking interval for the 3:2 subharmonic (i.e., $V_{1} / V_{2}=\frac{3}{2}$ ), for three values of the coupling $\alpha$. The other parameters are exactly those of the seriesaiding case of Refs. 9 and 11: $I_{1}=2, I_{c_{1}}=1.2, I_{c_{2}}=0.8, \delta_{1}=1$, and $\delta_{2}=\frac{2}{3}$. NS stands for numerical simulation and $\mathrm{PC}$ for perturbation calculation.

\begin{tabular}{ccccc}
\hline \hline & $\begin{array}{c}\text { Center of } \\
I_{2}\end{array}$ & Locking Interval & \multicolumn{2}{c}{$\begin{array}{c}\text { Length of } \\
\text { NS }\end{array}$} \\
\hline 0.05 & NS & PC & $\begin{array}{c}\text { Locking Interval } \\
\text { PC }\end{array}$ \\
0.07 & 1.816461 & 1.816461 & 0.000018 & 0.000022 \\
0.20 & 1.825910 & 1.825908 & 0.000035 & 0.000046 \\
\hline \hline
\end{tabular}

\section{NUMERICAL SIMULATION}

The equations which describe the system are, in appropriate units, ${ }^{9}$

$$
\begin{aligned}
& \frac{d \phi_{1}}{d t}=\delta_{1}\left(I_{1}-I_{c_{1}} \sin \phi_{1}\right)-\alpha\left(I_{2}-I_{c_{2}} \sin \phi_{2}\right), \\
& \frac{d \phi_{2}}{d t}=\delta_{2}\left(I_{2}-I_{c_{2}} \sin \phi_{2}\right)-\alpha\left(I_{1}-I_{c_{1}} \sin \phi_{1}\right),
\end{aligned}
$$

where $\phi_{1}, \phi_{2}$ are the phase difference across the weak links; $I_{c_{1}}, I_{c_{2}}$ are the corresponding critical currents, while $\alpha$ measures the coupling between junctions produced by various mechanisms such as quasiparticle diffusion ${ }^{10}$ or via a shunt resistor. ${ }^{9} \delta_{1}, \delta_{2}$ are a measure of the asymmetry of the junctions due to different resistances. $t$ is a dimensionless time as described in Ref. 9.

A fourth-order Runge-Kutta method was used in seventeen-decimal-digit arithmetic to solve the system (2). Great care was taken using extremely long-time runs to ensure that the short-time average $\left(n_{2} \phi_{2}-n_{1} \phi_{1}\right)$ was indeed

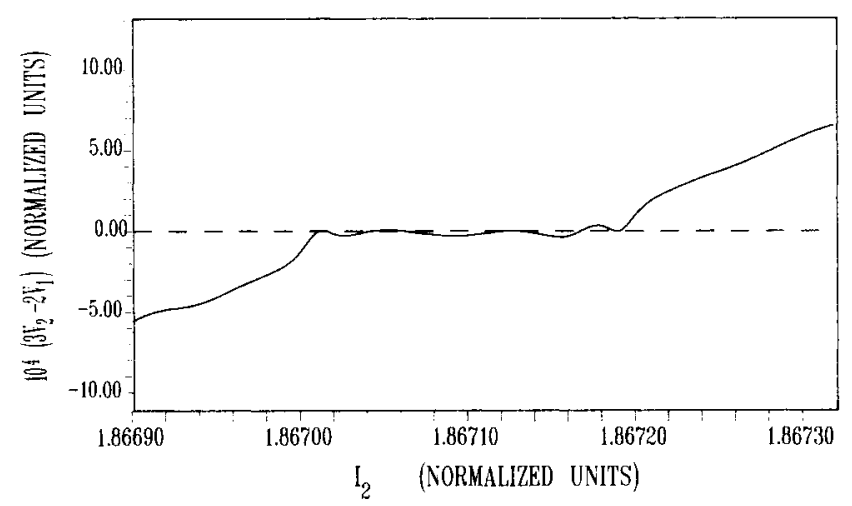

FIG. 1. An exploded view of the numerically determined $I-V$ curve in the vicinity of $3: 2$ locking for a pair of coupled Josephson weak links. Parameters are the same as for Table I, except we select the $\alpha=0.2$ case; i.e., this is exactly the case of the series aiding example of Refs. 9 and 11. $3 V_{2}-2 V_{1}$ is plotted so that the locking zone is the zero of this variable. Small fluctuations in the graph are due to the rapid oscillation $\sim 2 \pi$ which exists in the corresponding time-dependent voltage. These curves were determined by averaging over a time span of 120000 time units. (The end points of the zone as given in Table I were determined over even much longer runs.) constant in the locking zone. All but one parameter were held constant at typical values while $I_{2}$ was used as the control parameter, in exact accordance with the seriesaiding case associated with earlier practice. $9,11,12$

In particular the 3:2 subharmonic was selected for detailed consideration. Figure 1 shows the appropriate $I-V$ curve in the neighborhood of 3:2 locking illustrating the latter's continuous but rapid onset. Detailed determination of the $I_{2}$ locking interval was made for three values of $\alpha$. These intervals are listed together with the perturbation results in Table $I$. The comparison will be made below.

\section{PERTURBATION ANALYSIS}

As in previous work ${ }^{9-11}$ the analytic treatment of the system (2) depends upon treating the coupling parameter $\alpha$ as "small". We employed the method of averaging ${ }^{15}$ as we had done in Ref. 11, where, in order to implement the technique, we described a necessary variable change from $\left(\phi_{1}, \phi_{2}\right)$ to $\left(\xi_{1}, \xi_{2}\right)$ therein defined. Applying this process here and fixing all parameters except say $I_{2}$, subharmonic dc voltage locking $n_{2} V_{2}=n_{1} V_{1}$ will then occur for those $I_{2}$ which cause the time average

$$
\lim _{T \rightarrow \infty} \frac{1}{T} \int_{0}^{T} \frac{d}{d t}\left(n_{1} \xi_{1}-n_{2} \xi_{2}\right) d t
$$

to vanish. ${ }^{11} V_{1,2}$ are the respective dc (or average) voltages of links 1 and 2 while $n_{1}, n_{2}$ are positive relatively prime integers, neither of which is unity.

It follows that it is convenient to introduce new variables when studying a particular $n_{2}: n_{1}$ locking zone by

$$
\xi=n_{1} \xi_{1}+n_{2} \xi_{2}
$$

and

$$
D=n_{1} \xi_{1}-n_{2} \xi_{2},
$$

where we can anticipate that $\xi$ will be a "fast" variable while $D$ will be "slow." We only give a bare sketch of the ensuing calculation, since it is an adaptation of a similar one carried out in Ref. 11. Since some of the symbols have lengthy definitions we refer the reader to this same source for any here undefined quantities.

In terms of $\xi$ and $D,(2)$ becomes 


$$
\begin{aligned}
& \frac{d \xi}{d t}=\Omega^{+}-\alpha\left[\Gamma^{+} \cos \left(q^{+} \xi+q^{-} D\right)+\sum_{j, r, s} n_{j} Z_{j r s}^{i}\right] \\
& \frac{d D}{d t}=\Omega^{-}-\alpha\left[\Gamma^{-} \cos \left(q^{+} \xi+q^{-} D\right)+\sum_{j, r, s} \epsilon_{j i} n_{j} Z_{j r s}^{i}\right],
\end{aligned}
$$

where

$$
\begin{aligned}
& \Gamma^{ \pm}=\left(n_{1} \gamma_{11}^{2} \pm n_{2} \gamma_{21}^{1}\right), q^{ \pm}=\left(n_{2} \pm n_{1}\right) / 2, \\
& \Omega^{ \pm}=\left(n_{1} F_{1} \pm n_{2} F_{2}\right), \\
& q_{r s}^{+j l}= {\left[n_{j}(2 r+l+s) \pm n_{i} s\right] / 2, } \\
& F_{j}=\Omega_{j}+\alpha \sigma_{j}^{i} \cosh \lambda_{j}, \\
& Z_{j r s}^{i}=e^{-\lambda_{i} J_{j r s}^{i} \cos \left(q_{r s}^{-j 2} \xi+\epsilon_{i j} q_{r s}^{+j 2} D\right)} \\
& \quad+L_{j r s}^{i} \sin \left(q_{r s}^{-j 1} \xi+\epsilon_{i j} q_{r s}^{+j 1} D\right)
\end{aligned}
$$

where the summations in (5) are over

$$
r=0,1,2, \ldots, s=-1,0,1, i, j=1,2,
$$

but with $i \neq j$ and $\epsilon_{12}=-\epsilon_{21}=1$.

The Krylov-Bogoliubov-Mitropolski technique $e^{15}$ is applied to $(5)$ involving final change of variable to $(\bar{\xi}, \bar{D})$ where

$$
\begin{aligned}
& \xi=\bar{\xi}+\alpha \psi_{1}(\bar{\xi}, \bar{D})+\alpha^{2} \psi_{2}(\bar{\xi}, \bar{D})+O\left(\alpha^{3}\right), \\
& D=\bar{D}+\alpha \sigma_{1}(\bar{\xi}, \bar{D})+\alpha^{2} \sigma_{2}(\bar{\xi}, \bar{D})+O\left(\alpha^{3}\right),
\end{aligned}
$$

leading to the differential equations

$$
\begin{aligned}
& \frac{d \bar{\xi}}{d t}=\Omega^{+}+\alpha B_{1}(\bar{D})+\alpha^{2} B_{2}(\bar{D})+O\left(\alpha^{3}\right), \\
& \frac{d \bar{D}}{d t}=\Omega^{-}+\alpha A_{1}(\bar{D})+\alpha^{2} A_{2}(\bar{D})+O\left(\alpha^{3}\right) .
\end{aligned}
$$

The equations determining $A_{i}, B_{i}, \psi_{i}$, and $\sigma_{i}$ are obtained by inserting (7) and (8) into (5) and identifying like terms in $\alpha$. As part of the process $A_{i}$ and $B_{i}$ are chosen so that $\psi_{i}$ and $\sigma_{i}$ become periodic functions in $\bar{\xi}$ with zero average value.

It is readily shown that in this case $A_{1}=0=B_{1}$. Hence subharmonic locking is associated with second-order terms in $\alpha$, not first order as is the case of equal-voltage locking or harmonic locking., 9,11 Thus from the second equation of (8) we see that the criterion for subharmonic locking is to second order in $\alpha$ : $\Omega^{-}+\alpha^{2} A_{2}(\bar{D})=0$, the real roots of which (if they exist) as a function of $I_{2}$ determine the locking interval. The coefficients $A_{2}(\bar{D})$ and $B_{2}(\bar{D})$ are periodic functions of $\bar{D}$. (The algebraic details involved in the determination of $A_{2}$ and $B_{2}$ are very extensive and for brevity are omitted.)

The fact that first-order terms in $\alpha$ vanish implies that the width of the locking zone will be quadratic in $\alpha$, and hence much more difficult to observe for weak coupling than equal voltage or harmonic locking, which are mediated by a locking zone of order $\alpha .9,11$

Finally, it should be noted that a telescoping or "renormalization" process which effectively redefines the frequency, first introduced in Refs. 9 and 11 was again employed. This was done by choosing the $\lambda_{j}$ in (6) so that the $\sigma_{j}^{i}$ are zero and hence $F_{j}=\Omega_{j}$. In this context as in that of Ref. 11, it is not essential for the removal of secular terms as it had been in Ref. 9, but it does improve the accuracy of the results considerably, especially in regard to the position of the locking zone.

\section{RESULTS AND DISCUSSION}

Both the numerical simulation and the analytical perturbation calculation predict subharmonic locking for this system. We studied in detail the 3:2 subharmonic locking interval in $I_{2}$, for three values of the coupling constant $\alpha$. The results are shown in Table I. A graphical display for one case is given by the $I-V$ curves in Fig. 1.

As expected from the second order of the perturbation calculation the predicted locking intervals are extremely small at low coupling. For the two lower values of $\alpha$ the analytical calculation is in remarkable agreement with the "exact" numerical simulation, most especially in the predicted location of the locking interval, but also in the width of this interval. The renormalization or telescoping process mentioned above in regard to the perturbation theory is in good part responsible for the excellent positioning of the center of the interval. For the largest value of $\alpha$, of 0.2 , the quantitative agreement is no longer good, indicating the breakdown of second-order perturbation theory for this process. The numerical simulation itself indicates that the length of the interval is growing more slowly than $\alpha^{2}$ at $\alpha=0.2$, predicting the loss of validity of the second-order calculation.

In the vicinity of a particular subharmonic locking zone, perturbation theory, to a high degree of accuracy, distills Eqs. (5)-(8) down to an equation governing the appropriate average phase difference $\bar{D}$,

$$
\frac{d \bar{D}}{d t}=\alpha^{2}(A+B \sin \bar{D})
$$

where $A$ and $B$ are functions of the parameters of the system, $A$ vanishing precisely at the center of the locking zone. This simplified equation predicts that the interval of locking will be a mapping of the interval $-\pi / 2 \leqq \bar{D} \leqq \pi / 2$ onto the appropriate $I_{2}$ interval. That is, at the extreme of the locking zone where $A=B$ the average phase difference will be $-\pi / 2$, while when $A=-B$ at the other extreme, $\bar{D}=\pi / 2$.

In close analogy with our discussions of "voltage pulling" near equal voltage locking,, 11 one would expect from this equation partially coherent behavior just outside the subharmonic locking interval. That is when $|A|$ is greater than $|B|$ but approximately equal to $|B|$, one expects long periods of almost constant average $n_{2}: n_{1}$ phase differences interrupted by rapid slippages of $2 \pi$. This was observed in the numerical simulation and was used as a guide to "zeroing in" on the locking zone. It could be observed experimentally as well by observing partially coherent behavior outside the actual locking zone.

In conclusion we predict that mutual subharmonic volt- 
age locking of noncapacitive coupled weak links could be observed experimentally, practically speaking at least, for the lower values of $n_{2}, n_{1}$ and for strong enough coupling. This contrasts with the single ac driven noncapacitive weak link where no such locking is possible, regardless of the strength of the ac source.

\section{ACKNOWLEDGMENTS}

We thank Dr. J. T. Smith for useful discussion on the experimental aspects of this problem. This work was supported by grants from the Natural Science and Engineering Research Council of Canada.
${ }^{1}$ J. Bindslev Hansen and P. E. Lindelof, Rev. Mod. Phys. 56, 431 (1984).

2J. R. Waldram, A. B. Pippard, and J. Clarke, Philos. Trans. R. Soc. London, Ser. A 268, 265 (1970).

${ }^{3}$ M. J. Renne and D. Polder, Rev. Phys. Appl. 9, 25 (1978).

4J. R. Waldram and P. H. Wu, J. Low Temp. Phys. 47, 363 (1982).

${ }^{5}$ D. C. Cronemeyer, C. C. Chi, A. Davidson, and N. F. Pedersen, Phys. Rev. B 31, 2667 (1985).

${ }^{6} \mathrm{E}$. Rossler, in Nonlinear Oscillations in Biology, edited by F. C. Hoppinsteadt (American Mathematical Society, Providence, 1979).

${ }^{7}$ M. A. H. Nerenberg, James. A. Blackburn, and S. Vik, Phys. Rev. B 30, 5084 (1984).

${ }^{8}$ See, for example, R. L. Kautz, IEEE Trans. Magn., MAG-19,
465 (1983).

${ }^{9}$ M. A. H. Nerenberg, James. A. Blackburn, and D. W. Jillie, Phys. Rev. B 21, 118 (1980).

${ }^{10}$ D. W. Jillie, M. A. H. Nerenberg, and J. A. Blackburn, Phys. Rev. B 21, 125 (1980).

${ }^{11}$ A. S. Deakin and M. A. H. Nerenberg, Phys. Rev. B 25, 1559 (1982).

${ }^{12}$ A. S. Deakin and M. A. H. Nerenberg, Phys. Rev. B 27, 6954 (1983).

${ }^{13}$ M. A. H. Nerenberg and J. A. Blackburn, Phys. Rev. B 23, 1149 (1981).

${ }^{14}$ A. K. Jain, K. K. Likharev, J. E. Lukens, and T. E. Sauvageau, Phys. Rep. 109, 309 (1984).

${ }^{15}$ A. Nayfeh, Perturbation Methods (Wiley, New York, 1973). 